\title{
A Simple Expert System Shell for Microcomputer-Aided Radiographic Diagnosis
}

\author{
Morgan G. Dunne and Sean B. Dunne
}

\begin{abstract}
A simple microcomputer-based expert system shell for radiographic diagnosis is described. The system interrogates the user by means of a simple questionand-answer approach on the radiographic findings in a given case and proffers a differential diagnosis from a database. The system also allows listing of both the radiographic findings pertinent to a specific diagnosis and all diagnoses in which a particular finding or combination of findings occur. The system database can be easily updated or modified by the user. The demonstration application for the expert system shell is the plain film diagnosis of osseous dysplasia.
\end{abstract}

(C) 1990 by W.B. Saunders Company.

KEY WORDS: computers, software, expert systems, radiology, artificial intelligence, osseous dysplasia, bone diseases.

$\mathbf{T}$ HE USE OF personal computers (PC) and expert systems as an aid in radiographic diagnosis is being reported with increasing frequency. ${ }^{1-4}$ Many of these systems are complex, memory-expensive, and require mainframe or minicomputer power. The system described here was developed to acquire an introductory level understanding of expert system programming on a microcomputer (PC) and apply such programming to a specific problem in diagnostic radiology.

Differential diagnosis of the osseous dysplasias remains a problem for the general radiologist, as many of these conditions are rare and the diagnostic criteria are both numerous and nonspecific. It was felt that the interpretation of radiographs on these patients would be facilitated by the application of a simple user-friendly expert system running on a typical microcomputer $(\mathrm{PC})$, which would pose a series of questions regarding specific radiographic findings to be answered by using only three keys - "y" for "yes," " $n$ " for "no," or "d" for "don't know."

From the Department of Radiology, Baptist Memorial Hospital System, San Antonio, TX.

Address reprint requests to Morgan G. Dunne, $M A, M B$, $B C h, B A O, 15301$ Winter Mist, San Antonio, TX 78247.

(C) 1990 by W.B. Saunders Company.

0897-1889/90/0301-0004\$03.00/0

\section{PROGRAMMING LANGUAGE}

The programming language selected for the system development was TURBO PROLOG version 1.1 (Borland International, Scotts Valley, CA), which is a compiler-based implementation of PROLOG, a fifth-generation artificial intelligence language ideally suited to expert system and database design., ${ }^{5,6}$ The programming environment offered by TURBO PROLOG is quite easy to use with a word-processing style text editor, and provides extensive debugging aids that quickly locate and display errors in the source code for correction. Once compiled, the program runs at interactive speeds on an IBM PC-XT (IBM Corporation, Boca Raton, FL) even during extensive database analysis, in contrast to other relational database programs. The PROLOG language is a declarative rather than procedural language, meaning that it attempts to solve a problem by deductive reasoning given a set of facts and rules. Thus, the programmer has only to provide a description of the problem and general set of rules for solving it and the PROLOG system will do the rest.

\section{EXPERT SYSTEM}

The expert system consists of two parts, the knowledge base (KB) and the inference engine (IE) ${ }^{7,8}$ The KB is a database that holds a list of objects (eg, osseous dysplasia diagnoses) and their associated rules (lists of incompatible radiographic findings) and attributes (radiographic findings pertaining to a particular diagnosis). The KB may reside on a disk and be accessed as needed by the program, or it may be incorporated into the program code as was done in this case to allow the program to run faster without the need for pauses while the disk is read. The KB predicates and clauses contain a descriptor that indicates to which application they belong ("OD" in this case for osseous dysplasia). Thus, the IE is designed to operate with other user-selected databases, which may be either maintained on a disk or embedded within the program. ${ }^{8}$ Each radiographic finding clause in the KB is numbered and the diagnosis clauses contain a list of integers designating the associated radiographic findings. 
The data on the 106 diagnoses of osseous dysplasia for the demonstration KB were obtained from standard sources. ${ }^{9-12}$

The IE is the part of the program that attempts to find a matching diagnosis given the radiographic findings which are determined to be present or absent based on the user's responses to the questions generated by the system. The IE used in this application is deterministic. ${ }^{7}$ This means that it will only proffer a diagnosis if the radiographic findings associated with that diagnosis are either confirmed by the user or the user indicates that he or she does not know whether or not some or all of the findings associated with that diagnosis are present. The IE in this system uses the backward-chaining or object-driven method. ${ }^{7,8}$ In this method, the program assumes a hypothesis which it attempts to prove by taking the first object (diagnosis) in the database and asking the user if each of the findings associated with that diagnosis are present. If any of the findings are indicated by the user not to be present (signified by pressing the " $n$ " key), then the IE will reject the diagnosis and move on to the next diagnosis and repeat the process. If a given finding is given a "don't know" answer by the user (signified by pressing the " $d$ " key), then the IE assumes that finding is present and remains with the diagnosis in question and continues to ask questions about the other findings associated with the diagnosis.

The IE maintains two memory resident databases (MRDs) which keep track of the "yes" and "no" responses so that the user is not asked the same question twice. "Don't know" answers are stored as "yes" responses. Thus, when each diagnostic hypothesis is picked up by the IE, the associated radiographic findings are checked in turn against the MRDs to determine if a "yes" or "no" response has already been elicited for that finding. If a given finding is found in the "no" MRD, then the diagnostic hypothesis in question is rejected and the next diagnosis in the database is placed under consideration. If the finding is found in the "yes" MRD, then the IE moves on to the next finding associated with that diagnosis and repeats the process. If the finding is not found in either of the "yes" or "no" MRDs, then the user is questioned as to whether or not the finding is present.
The IE contains three rule-based subroutines, each with an associated database written into the program which contains a specific subset of radiographic findings. The purpose of these subroutines is to reduce the number of questions that the user must answer in order to obtain a complete differential diagnosis. The first of these deals with normalcy in a specific anatomic area. For example, if the user's response is "yes" to the question "normal skull and facial bones?", then all radiographic findings relating to the skull and facial bones are appended to the "no" MRD. Thus, if the skull and facial bones are normal in a given case, the user is not asked any further questions regarding this anatomic area. The second subroutine deals with specific interrelated and incompatible radiographic findings. For example, if the user confirms the presence of micrognathism, then the finding of prognathism is appended to the "no" MRD. The third subroutine deals with opposite and incompatible radiographic findings. For example, if the user enters " $n$ " to the question "hemangiomata present?", then the finding "hemangiomata absent" is appended to the "yes" MRD.

\section{USER INTERFACE}

The user interface consists of several menucontaining windows that are called to the screen according to keyboard input. The main menu (Table 1) consists of five choices, the first of which activates the interactive expert system and IE leading to a differential diagnosis based on a thorough interrogation of the user through a "diagnostic findings" window. Each question is displayed on the screen and the user enters "y," " $n$," or " $d$." The user's response is displayed in full after each question (Table 2). Each time the IE establishes a diagnosis the screen switches to a "differential diagnosis" window and the diagno-

Table 1. System Main Menu

ODLOG.PRO: Osseous Dysplasia Logic-Based Expert System

Welcome to ODLOG.PRO !

1. Consult Expert System

2. List All Diagnoses on Knowledge Base

3. List Findings for Specific Diagnosis

4. List Diagnoses with Specific Finding(s)

5. Exit Expert System

Please enter your choice: 1-5 
Table 2. Typical Dialogue With the Expert System

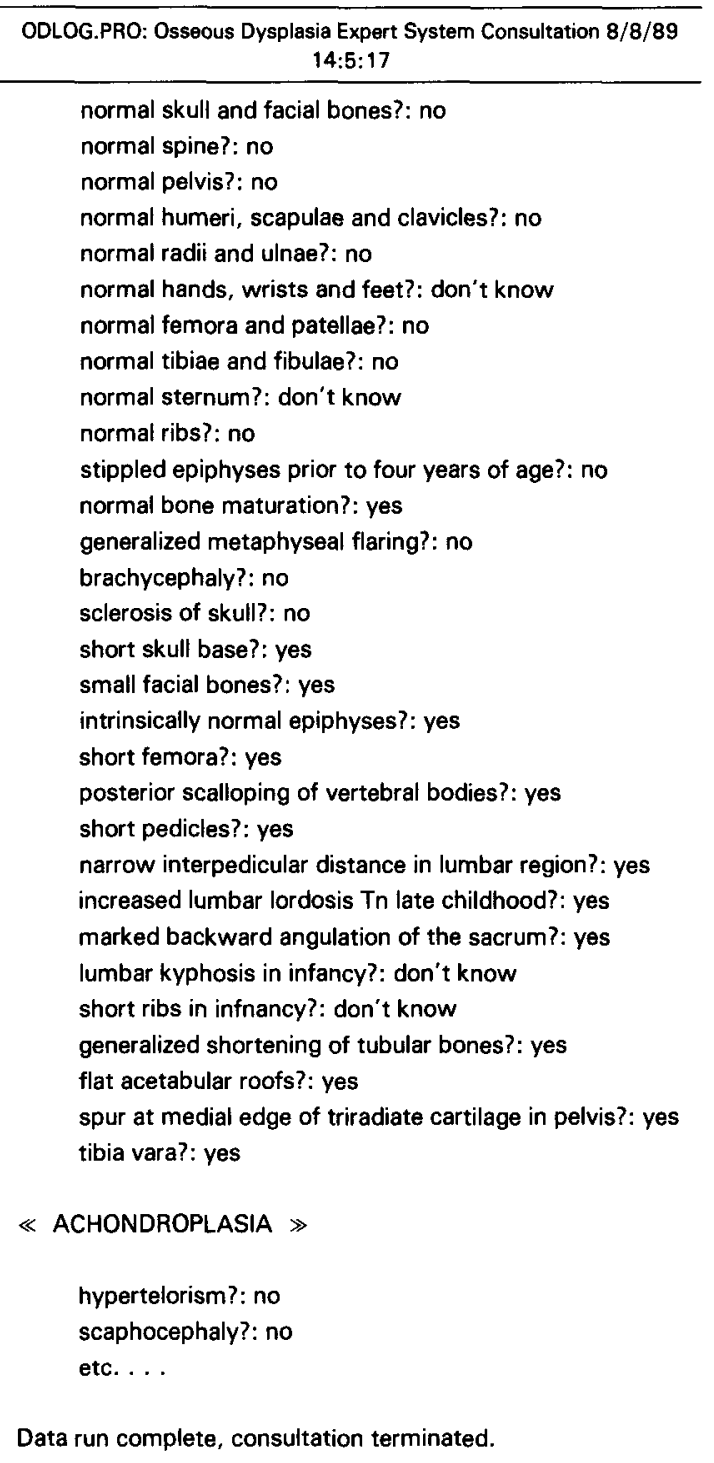

sis or diagnoses are displayed. The user returns to the diagnostic findings window by pressing the spacebar and the interrogation resumes as the IE searches for additional diagnoses.

The second choice from the main menu simply lists all the diagnoses contained in the database (106 at the time of this writing), one screenful at a time. The third menu choice allows the user to select a specific diagnosis from a submenu and view all the radiographic findings associated with that diagnosis (Table 3). The fourth menu choice allows the user to select one or more radiographic findings from a submenu and have the system display all diagnoses on the database that are associated with those findings (Table 4). Thus, for example, the system could list all diagnoses
Table 3. List of Findings for Specific Diagnosis

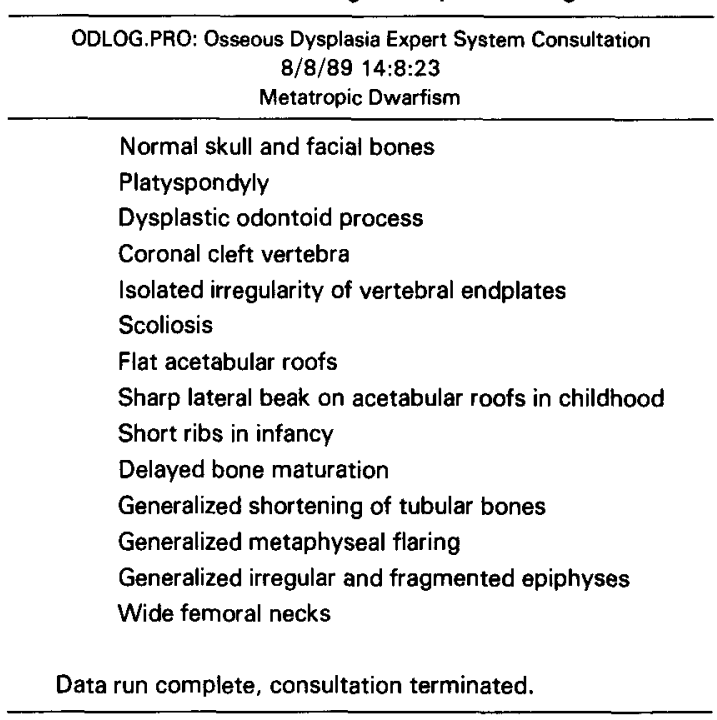

associated with platyspondyly and generalized tubular bone shortening (Table 4). The fifth choice on the main menu exits the system.

\section{HARD COPY}

Printed hard copy can be obtained from any of the first four main menu choices. The system accomplishes this by opening a diskfile when one of the first four choices is selected. Everything that is displayed on the screen (except menus) is also written in this file and at the end of each consultation the user is offered the option of printing this file or simply returning to the main menu. An example of a printout of a typical dialogue with the expert system is shown in Table 2. At the end of each consultation the diskfile is

Table 4. List of Diagnoses With Specific Finding(s)

ODLOG.PRO: Osseous Dysplasia Expert System Consultation 8/8/89 14:11:12

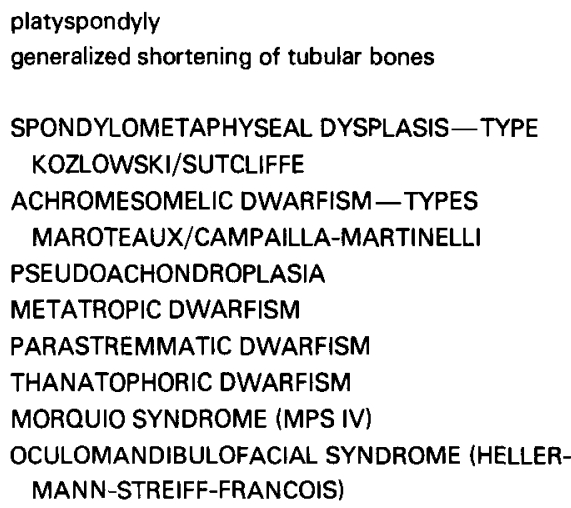

Data run complete, consultation terminated. 
erased. Each printout is stamped with the date and time of the consultation.

\section{KNOWLEDGE ENGINEERING}

Perhaps the most difficult aspect of expert system development is the organization of the knowledge database for optimum efficiency. Ideally, one would like the system to provide a list of differential diagnoses in descending order of probability, asking the user as few questions as possible. In order to develop this ideal system a probabilistic rather than deterministic IE would be required and the knowledge base would need to contain hard data regarding the prevalence and incidence of the various diagnoses, discriminant criteria for the radiographic findings associated with a given diagnosis, and positivity criteria for each of the various radiographic findings. The sensitivity and specificity of the radiographic test (plain film in this case) also would need to be included in the database. ${ }^{13}$ Because such hard data is rarely if ever available in those problems relating to diagnostic radiology, one is forced to settle for a deterministic system as described previously or to develop one's own scoring system to indicate the likelihood of each of the diagnoses in the differential. ${ }^{14}$ The diagnoses in the system described previously can be arranged in the database in descending order of likelihood according to the user's practice. By this means the IE will attempt to confirm or reject the more common diagnoses first and the least likely diagnoses last.

The inclusion or deletion of specific radiographic findings from the database is a potentially subjective undertaking because the positivity criterion for a particular finding may vary from one radiologist to another. Thus, a given radiologist user may either find that a particular finding either does not cross his or her threshold sufficiently of ten to allow reasonable sensitivity in making a diagnosis or may feel that the finding is not particularly specific. ${ }^{13}$ Because the absence of a particular finding is crucial in the rejection of a diagnosis in the system described previously, it was felt necessary to include the "don't know" option to allow the user a way of handling uncertainty regarding a particular finding in a given case. Until more experience is gathered in the clinical setting with the system described and similar systems, we must await the establishment of an "official" set of radiographic findings for application to a particular radiologic problem. The approach used in the system described was to include initially a large number of findings (250 at time of this writing) and hopefully whittle these down to a smaller, more discriminant number as experience with the system is acquired. For example, the finding of scoliosis, while a prominent feature of many osseous dysplasias, is not particularly useful in their differentiation as it may occur in isolation or be associated with almost any spinal anomaly. On the other hand, and hypothetically, if scoliosis never occurred in a specific dysplasia, then its inclusion in the database would be of value as its absence in a given case may help confirm a diagnosis.

\section{SYSTEM REQUIREMENTS}

The TURBO PROLOG environment requires at least $384 \mathrm{~K}$ of RAM and the stack size should be set to 2,400 paragraphs. The program source code takes up $14 \mathrm{~K}$, and $26 \mathrm{~K}$ was required for the $\mathrm{KB}$ at time of writing. Thus, the entire system takes $40 \mathrm{~K}$ with the $\mathrm{KB}$ embedded in the program. An option programmed into the user interface, but not yet added to the KB, is the ability of the system to display an explanation of the radiographic finding while being questioned by the IE. The finding explanation window is accessed by pressing the " $\mathrm{e}$ " key in response to a question by the system and the interrogation is resumed by pressing the spacebar after the explanation has been read by the user. The addition of explanations for all radiographic findings probably will not be required, as many are self explanatory. Nevertheless the memory requirements for the KB is expected to increase to approximately $50 \mathrm{~K}$ with the addition of these data.

The expert system shell described offers a fast, inexpensive approach to computer-aided diagnosis of certain specific radiologic problems. It may be used as a simple database manager without activating the IE. The powerful word processing ability of the TURBO PROLOG editor allows rapid deletion or addition of diagnoses and radiographic findings to the database by the user. With increasing introduction of computer technology to the radiology department it may behoove the practicing radiologist to become more familiar with medical expert systems and terminology. It is hoped that with increased input from such individuals systems with enhanced practical clinical usefulness will be developed. 


\section{REFERENCES}

1. Greenes RA: Computer-aided diagnostic strategy selection. Radiol Clin North Am 24:105-120, 1986

2. Kahn CE, Messersmith RN, Jokich MD: PHOENIX: An expert system for selecting diagnostic imaging procedures. Invest Radiol 22:978-980, 1987

3. Swett HA, Miller PL: ICON: A computer-based approach to differential diagnosis in radiology. Radiology 163:555-558, 1987

4. Swett HA, Fisher PR, Cohn AI, et al: Expert systemcontrolled image display. Radiology 172:487-493, 1989

5. Schildt H: Artificial intelligence: A quick overview, in Schildt H: Advanced Turbo Prolog ${ }^{\circledR}$. Berkley, CA, Osborne/ McGraw-Hill, 1987, pp 1-12

6. Yin KM, Solomon D: Introduction, in Yin KM, Solomon H (eds): Using Turbo Prolog ${ }^{\oplus}$. Indianapolis, IN, Que Corporation, 1987, pp 1-6

7. Schildt $H$ : Artificial intelligence: Expert systems, in Schildt H: Advanced Turbo Prolog ${ }^{\circledR}$. Berkley, CA, Osborne/ McGraw-Hill, 1987, pp 57-90

8. Yin KM, Solomon D: Creating expert systems, in Yin
KM, Solomon D: Using Turbo Prolog ${ }^{\otimes}$. Indianapolis, IN, Que Corporation, 1987, pp 387-434

9. Wynne-Davies R, Hall CM, Apley AG: Atlas of Skeletal Dysplasias. Edinburgh, Scotland, Churchill Livingstone, 1985

10. Taybi H: Radiology of Syndromes and Metabolic Disorders (ed 2). Chicago, Year Book Medical, 1983

11. Goldman AB: Collagen diseases, epiphyseal dysplasias, and related conditions, in Resnik D, Niwayama G (eds): Diagnosis of Bone and Joint Disorders, vol 5. Philadelphia, PA, Saunders, 1988, pp 3374-3441

12. McAlister WH: Osteochondrodysplasias, dysostoses, chromosomal aberrations, mucopolysaccharidoses, and mucolipidoses, in Resnik D, Niwayama G (eds): Diagnosis of Bone and Joint Disorders, vol 5. Philadelphia, PA, Saunders, 1988, pp 3442-3515

13. Chang P: Bayesian analysis revisited: A radiologist's survival guide. AJR 152:721-727, 1989

14. Getty DJ, Pickett RM, D’Orsi CJ, et al: Enhanced interpretation of diagnostic images. Invest Radiol 23:240252,1988 